\title{
Upregulation of antioxidant nuclear factor erythroid 2- related factor 2 and its dependent genes associated with enhancing renal ischemic preconditioning renoprotection using levosimendan and cilostazol in an ischemia/reperfusion rat model
}

\author{
Mona K. Tawfik ${ }^{1}$, Samy Makary², Mohammed M. Keshawy ${ }^{3}$
}

\begin{abstract}
${ }^{1}$ Department of Pharmacology, Faculty of Medicine, Suez Canal University, Ismailia, Egypt ${ }^{2}$ Department of Physiology, Faculty of Medicine, Suez Canal University, Ismailia, Egypt ${ }^{3}$ Department of Internal Medicine, Nephrology Division, Faculty of Medicine, Suez Canal University, Ismailia, Egypt
\end{abstract}

Submitted: 24 November 2018, Accepted: 28 July 2019,

Online publication: 28 January 2021

Arch Med Sci 2021; 17 (6): 1783-1796

DOI: https://doi.org/10.5114/aoms/111373

Copyright (c) 2021 Termedia \& Banach

\section{Abstract}

Introduction: Ischemic preconditioning (Ipre) provides protection against renal ischemia-reperfusion (I/R) injury with its associated remote organ damage. This study examined the enhancing protective effect of Ipre with levosimendan or cilostazol in I/R-induced kidney and lung injury in a rat model.

Material and methods: Rats were divided into: sham-operated, I/R control, Ipre control, I/R + cilostazol or levosimendan and Ipre + cilostazol or levosimendan. Drugs were given 30 min before left renal I/R or 4 cycles of Ipre just before renal ischemia.

Results: The Ipre combined with the implemented drugs enhanced physiological antioxidant defense genes including renal nuclear factor erythroid 2-related factor 2 (Nrf2) and its dependent genes heme oxygenase-1 (HO-1) and NADPH-quinone oxidoreductase-1 (NQO-1) and improved malondialdehyde and superoxide dismutase renal tissue levels. The combined effect improved I/R consequences for blood urea, creatinine, and creatinine clearance and improved blood oxygenation and metabolic acidosis. Moreover, the combination improved the renal soluble intercellular adhesion molecule (ICAM), tumor necrosis factor $\alpha$ (TNF- $\alpha$ ) and interlukin-6 (IL-6) with histopathological improvement of tubular necrosis with a decrease in the apoptotic marker caspase- 3 and an increase in the anti-apoptotic Bcl-2 expression.

Conclusions: Cilostazol or levosimendan potentiates the renoprotective effect of Ipre against renal I/R injury, associated with upregulation of antioxidant genes Nrf2, HO-1, and NOQ-1 expression.

Key words: ischemic preconditioning, renal ischemia reperfusion, levosimendan, cilostazol, nuclear factor-erythroid-2-related factor 2.

\section{Introduction}

Renal ischemia-reperfusion (I/R) injury is a substantial clinical problem in renal transplantation, which may cause renal dysfunction [1]. The pathophysiological mechanisms of I/R injury are associated with increased expression of intercellular adhesion molecules [2, 3] and pro-inflammatory

\author{
Corresponding author: \\ Mona K. Tawfik \\ Department of Pharmacology \\ Faculty of Medicine \\ Suez Canal University \\ Ismailia 41522, Egypt \\ Phone: +20 1222719473 \\ E-mail: \\ dmon_kamal@yahoo.com
}


cytokine [4], leading to acute kidney injury (AKI) [2, 3]. The disturbed intracellular adhesion molecule-1 (ICAM-1) with vascular adhesion molecule leads to activation and trapping of leucocytes. Local cytokines activate leucocytes, which initiate reactive oxygen species (ROS) production, which is a feature of kidney injury in $\mathrm{I} / \mathrm{R}$ and other chronic kidney diseases $[2,5]$. Subsequently, the whole processes end up with tissue swelling and vascular occlusion [2, 6] and enhanced apoptosis and cellular death [1]. Renal I/R injury subsequently leads to pulmonary failure $[2,7]$.

In contrast, ischemic preconditioning (Ipre) has renoprotective effects by suppressing apoptosis [8] and modulating the cellular cytokine release [9]. Induction of Ipre confers an intrinsic adaptive mechanism and protects against I/R-induced injury [10-12].

Nuclear factor-erythroid-2-related factor 2 (Nrf2), a transcription factor, and its target gene $\mathrm{NAD}(\mathrm{P}) \mathrm{H}$ :quinone oxidoreductase-1 (NQO-1) regulate antioxidant genes, affecting the expression of inducible heme oxygenase-1 (HO-1) [13, 14]. Controlling the inflammatory condition and oxidative stress was shown to have a protective effect through upregulating Nrf2, NQO-1 and $\mathrm{HO}-\mathrm{I}$ genes in ischemic tissues which reduce apoptosis $[15,16]$. The genes also showed a protective effect against chronic kidney diseases [17, 18].

Cilostazol is a calcium sensitizer known by its ROS scavenging effects $[19,20]$ and suppressing effect on the inflammatory cytokines and apoptotic protein expressions [21].

Levosimendan is another calcium sensitizer, which exhibits anti-aggregatory properties [22] and was shown to reduce AKI [23] through its antiinflammatory and ROS profiles [24].

As cilostazol and levosimendan were effective in ameliorating renal I/R [25-27], in the present study, we evaluated the protective effect of cilostazol or levosimendan, with and without Ipre, on I/R-induced renal injury and injury of other organs such as the lung. In renal I/R and Ipre, we explored the master regulator of antioxidant gene (Nrf2) expression, and the expression of the antioxidant gene NQO-1, which play a role in both $\mathrm{HO}-1$ expression and ROS production.

Thus, this study aims to demonstrate renal I/R pathophysiology, and the possible reno-protection therapeutic targets of cilostazol and levosimendan.

\section{Material and methods}

Seventy adult male albino rats weighing 240$260 \mathrm{~g}$ were used. A period of acclimatization was implemented as stated before [28]. The experiments were conducted according to the legal requirements in Poland as well as the National Institute of Health Guide (National Institute of Health
Publications No. 80-23, Revised 1978) for the care and use of laboratory animals for experimental procedure, and the institute ethical standards (ethics committee, No. 3324).

\section{Surgery and experimental protocol}

Rats were injected with pentobarbital $(50 \mathrm{mg} / \mathrm{kg} /$ i.p.), then Ipre and I/R protocols were applied according to Joo et al. [10]. Right nephrectomy was done first, followed by Ipre then I/R inductions. Renal Ipre was induced by placing a microvascular clamp on the left renal pedicle for 5 min, interrupted by 5 min reperfusion for 4 cycles, followed immediately by an I/R maneuver by subjecting the left renal pedicle to $30 \mathrm{~min}$ of ischemia and reperfusion for $48 \mathrm{~h}$.

Cilostazol and levosimendan (Sigma-Aldrich Chemical Co., Egypt) were supplied as white and crystalline powders, respectively, and dissolved in normal saline.

To test the effect of cilostazol as an ROS scavenger and levosimendan as anti-aggregatory or its combination in tissue protection, cilostazol (10 mg/kg, twice/day, oral gavage) was administered 2 h $[19,29]$, and levosimendan $(24 \mu \mathrm{g} / \mathrm{kg} /$ day, intraperitoneal) $30 \mathrm{~min}$ before the procedures [30, 31].

The animals were divided into seven groups of 10 animals each: (1) the sham group (baseline group): rats were subjected to left renal pedicle exposure with no ischemia, (2) I/R (ischemic reperfusion), (3) Ipre (Ipre + I/R), (4) I/R + cilostazol, (5) I/R + levosimendan, (6) Ipre + cilostazol, and (7) Ipre + levosimendan.

After $48 \mathrm{~h}$ of reperfusion, animals were euthanized under anesthesia by exsanguination. The blood samples and a portion of the left kidney and lung tissues were collected and kept at $-80^{\circ} \mathrm{C}$. The other part was embedded in $10 \%$ neutral buffered formalin for histopathological and immunohistochemical assays.

\section{Renal functions}

Renal dysfunction was evaluated to determine the effect of I/R, Ipre and the drugs by measuring serum levels of blood urea and creatinine using a Bioclin kit (Santa Coloma, Spain). Urine during $24 \mathrm{~h}$ was collected using metabolic cages, then creatinine clearance $(\mathrm{CrCl})$ was calculated as: $\mathrm{CrCl}=$ (urine creatinine; $\mathrm{mg}=\mathrm{dl} \times$ urine volume; $\mathrm{ml} / \mathrm{day}$ )/ (serum creatinine; $\mathrm{mg}=\mathrm{dl} \times 1440 \mathrm{~min}$ ) [11].

\section{Arterial blood gas analysis}

To study the effect on lung functions, arterial blood samples were obtained from abdominal aorta for $\mathrm{pH}$, partial pressure of oxygen $\left(\mathrm{PaO}_{2}\right)$, partial pressure of carbon dioxide $\left(\mathrm{PaCO}_{2}\right)$ and $\mathrm{HCO}_{3}^{-}$ 
measurements [32] with a blood sample analyzer (Roche Diagnostics OMNI C, IN, USA).

\section{Renal and lung oxidative stress levels}

Tissue lipid peroxides (LPS) of malondialdehyde (MDA) [6] and glutathione (GSH) levels were measured [28]. The activity of both superoxide dismutase (SOD) [6] and catalase (CATA) [19, 28] was also assessed to evaluate the impact of $I / R$ and the benefits of the Ipre and drugs.

\section{Renal and lung inflammatory assessment}

TNF- $\alpha$, IL- 6 and ICAM- 1 levels known to be increased with I/R were measured in both renal and lung homogenates using ELISA (BioSource Europe S.A., Brussels, Belgium).

\section{Histopathological evaluation}

Both renal and lung damage were evaluated and scored according to Azarkish et al. [7]. The kidney damage was assessed according to the presence of ischemic necrosis, tubular atrophy, hyaline casts and vacuolization. The lung tissue damage was assessed for the presence of congestion, inflammation, and fibrosis. The damage was scored from 1 to 4 , where 0 was assigned as normal tissue.

\section{Immunohistochemistry analysis}

Rat monoclonal antibodies against apoptotic markers (caspase-3-Bcl-2; Abcam, Cambridge, UK) in renal tissues were used to assess the deleterious effects associated with I/R. The percentage of immunopositive areas was determined using Image J $1.45 \mathrm{~F}$.

\section{Renal antioxidant genes expression}

Antioxidant Nrf2, NQO-1 andHO-1 gene expression is sensitive to I/R. Therefore, their levels were assessed from renal tissue, using quantitative real-time reverse transcription polymerase chain reaction (qRT-PCR) [33]. The gene-specific primer pairs were as follows: for Nrf2, forward primer 5'-ATCCAGACAGACACCAGTGGATC-3', reverse primer 5'-GGCAGTGAAGACTGAACTTTCA-3'. The (NQO-1) forward primer 5'-AGGCTGGTTTGAGCGAGT-3', reverse primer 5'-ATTGAATTCGGGCGTCTGCTG-3'; for $\beta$-actin, forward primer $5^{\prime}$-TGTTTGAGACCTTCAACACC-3', the reverse primer 5'-CGCTCATTGCCGATAGTGAT-3'. The (HO-1) forward primer 5'-TGCTCAACATCCAGCTCTTTGA-3', reverse primer 5'-GCAGAATCTTGCACTTTGTTGCT-3'.

\section{Statistical analysis}

Data were analyzed using SPSS, and expressed as mean \pm SD. The difference between variables was analyzed using one-way analysis of variance
(ANOVA) for quantitative variables, and Kruskal-Wallis test for parameters with non-Gaussian distribution, followed by Tukey's post-hoc test for multiple comparisons. A difference between groups was considered significant when $p$-value $<0.05$.

\section{Results}

Ischemic preconditioning and cilostazol or levosimendan enhanced renal and lung physiological parameters in ischemia-reperfusion

Renal I/R induced an elevation in the serum levels of urea and creatinine associated with a reduction in creatinine clearance $(\mathrm{CrCl})$ compared with the sham-operated group, suggesting I/R-induced renal dysfunction $(p<0.05$, Table I). Despite the functional $(p<0.05)$ recovery associated with the Ipre group in comparison with the I/R group, adding cilostazol or levosimendan to the Ipre group demonstrated more functional recovery $(p<0.05)$ when compared to the Ipre group. Ipre + levosimendan induced improvement compared to the Ipre + cilostazol treated group $(p<0.05$, Table I).

Rat arterial blood gas analysis was performed to further assess the consequences of renal injury and the protective effect of Ipre and the implemented drugs on lung injury (Table II). The arterial blood $\mathrm{pH}, \mathrm{PaO}_{2}$ and $\mathrm{PaCO}_{2}$ values in the Ipre + levosimendan group were improved $(p<0.05)$ compared to the Ipre and I/R groups.

\section{Ischemic preconditioning and cilostazol or levosimendan improved renal and lung oxidative stress and inflammatory cytokines}

I/R induced an oxidative stress burden associated with an increment of the inflammatory cytokines $(p<0.05)$ in both renal and lung tissue homogenates in comparison with the shamoperated group (Figures 1 and 2). Ischemic preconditioning either alone or in combination with the implemented pharmacological agents revealed effectiveness with varying degrees in ameliorating these deleterious effects associated with renal I/R. The Ipre + levosimendan treated group exhibited more improvement in these markers compared to the Ipre or Ipre + cilostazol group $(p<0.05)$ (Figures 1 and 2).

\section{Ischemic preconditioning and cilostazol or levosimendan improved renal and lung histopathological changes}

The kidney histopathological examination in the I/R group exhibited marked tubular degeneration in comparison with the normal sham group (Figure 3-I A, B). There is focal ischemic tubu- 
Table I. Effects of ischemic preconditioning, cilostazol or levosimendan and combination of them on renal function in renal ischemia/reperfusion injury

\begin{tabular}{|c|c|c|c|}
\hline Groups & $\begin{array}{l}\text { Serum creatinine } \\
{[\mathrm{mg} / \mathrm{dl}]}\end{array}$ & $\begin{array}{l}\text { Serum urea } \\
{[\mathrm{mg} / \mathrm{dl}]}\end{array}$ & $\begin{array}{l}\text { Creatinine clearance } \\
{[\mathrm{ml} / \mathrm{min}]}\end{array}$ \\
\hline Sham & $0.61 \pm 0.1$ & $26.1 \pm 2.6$ & $1.31 \pm 0.23$ \\
\hline $\mathrm{I} / \mathrm{R}$ & $2.5 \pm 0.15^{\prime \prime}$ & $73.8 \pm 7.8^{4}$ & $0.13 \pm 0.03^{\prime \prime}$ \\
\hline Ipre & $2.1 \pm 0.17^{\star}$ & $62.0 \pm 7.4^{\star 5}$ & $0.46 \pm 0.09^{\mho * \$}$ \\
\hline I/R + cilostazol & $1.8 \pm 0.13^{*}$ & $56.8 \pm 3.9^{\star \$}$ & $0.63 \pm 0.09^{4 \times \$}$ \\
\hline I/R + levosimendan & $1.65 \pm 0.25^{* \#}$ & $49.1 \pm 8.1^{\star \# \#}$ & $0.68 \pm 0.11^{1 *}$ \\
\hline Ipre + cilostazol & $1.33 \pm 0.11^{* \#}$ & $45.2 \pm 4.5^{\star \#}$ & 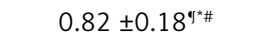 \\
\hline Ipre + levosimendan & $0.88 \pm 0.09^{* \# 5}$ & $35.1 \pm 3.9^{* \# \$}$ & $1.08 \pm 0.04^{* \# \$}$ \\
\hline
\end{tabular}

$I / R$ - ischemia/reperfusion, Ipre - ischemic preconditioning. Renal functions were assessed by serum creatinine, urea, and creatinine clearance. Results are mean $\pm S D(n=8-10)$ and analyzed using one-way ANOVA followed by Tukey's post-hoc test at $p<0.05$. "Compared to sham group. "Compared to I/R group. "Compared to Ipre group. $\$$ Ipre + cilostazol vs. Ipre + levosimendan.

Table II. Effects of ischemic preconditioning, cilostazol or levosimendan and combination of them on arterial blood gas analysis in renal ischemia/reperfusion injury

\begin{tabular}{|c|c|c|c|c|}
\hline Groups & $\mathrm{pH}$ & $\begin{array}{c}\mathrm{PaO}_{2} \\
{[\mathrm{~mm} \mathrm{Hg}]}\end{array}$ & $\begin{array}{c}\mathrm{PaCO}_{2} \\
{[\mathrm{~mm} \mathrm{Hg}]}\end{array}$ & $\begin{array}{c}\mathrm{HCO}_{3}^{-} \\
{\left[\mathrm{mEq}^{-} \mathrm{ll}\right]}\end{array}$ \\
\hline Sham & $7.35 \pm 0.02$ & $121.4 \pm 2.75$ & $38.20 \pm 1.27$ & $31.25 \pm 2.97$ \\
\hline I/R & $7.17 \pm 0.03^{4}$ & $92.55 \pm 2.71^{9}$ & $29.20 \pm 1.48^{9}$ & $16.88 \pm 3.03^{\prime \prime}$ \\
\hline Ipre & $7.19 \pm 0.03^{4}$ & $93.40 \pm 1.43^{\circ}$ & $29.47 \pm 1.39^{9}$ & $18.18 \pm 2.54^{\prime \prime}$ \\
\hline I/R + cilostazol & $7.21 \pm 0.02^{\triangleleft}$ & $96.05 \pm 1.24^{4}$ & $29.07 \pm 1.69^{4}$ & $17.00 \pm 2.56^{\prime \prime}$ \\
\hline I/R + levosimendan & $7.24 \pm 0.03^{9^{*}}$ & $98.23 \pm 2.24^{J^{\star}}$ & $31.02 \pm 1.79^{9}$ & $19.63 \pm 3.46^{\prime \prime}$ \\
\hline Ipre + cilostazol & $7.23 \pm 0.04^{\Psi^{\star}}$ & $106.8 \pm 2.89^{\sqrt{*} \#}$ & $30.43 \pm 2.15^{\circ}$ & $20.87 \pm 3.38^{4}$ \\
\hline Ipre + levosimendan & $7.35 \pm 0.02^{4 * \# \$}$ & $111.9 \pm 4.71^{\mathrm{y}^{\star} \# \$}$ & $34.62 \pm 0.66^{\sqrt{*} \# 5}$ & $26.50 \pm 3.38^{* \# 5}$ \\
\hline
\end{tabular}

$I / R$ - ischemia/reperfusion, Ipre - ischemic preconditioning. Data are expressed as mean $\pm S D(n=8-10)$ and analyzed using one-way ANOVA followed by Tukey's post-hoc test at $p<0.05$. "Compared to sham group. "Compared to I/R group. "Compared to Ipre group. ${ }^{5} / p r e$ + cilostazol vs. Ipre + levosimendan.

lar necrosis with shrunken glomeruli indicating ischemic necrosis (Figure 3-I B) with an increase $(p<0.05)$ in the mean histopathological score (Figure 3-II) compared to the sham group. Ischemic preconditioning + the implemented pharmacological agents ameliorated I/R effects (Figure 3-I) compared to the Ipre or I/R group (Figure 3-II; $p<0.05)$. Parallel damaging changes in the lung tissue was observed (Figure 4) in rats with I/R. Notably, Ipre slightly protected against disrupted alveolar structure, pulmonary interstitial edema and inflammatory cells in the alveolar cavity. However, Ipre + levosimendan had a greater protective effect in lung tissue (Figure 4-II).

\section{Ischemic preconditioning and cilostazol or levosimendan improved renal apoptotic changes}

Figures 5 and 6 show the enhanced renal apoptosis $(p<0.05)$ in I/R renal tissues as revealed by the elevated pro-apoptotic activator "caspase-3" percentage (Figure 5) and the declined anti-apoptotic protein "Bcl-2" (Figure 6) compared to the sham group.
Ipre, cilostazol or levosimendan and the combination of them inhibited apoptosis when compared to the I/R control group (Figures 5 and 6). The Ipre + levosimendan group displayed a reduction in caspase-3 and an elevation in $\mathrm{Bcl}-2$ as compared to the Ipre or Ipre + cilostazol group $(p<0.05)$ (Figures 5-II, 6-II).

\section{Ischemic preconditioning and cilostazol or levosimendan enhanced antioxidant genes}

Renal expression of Nrf2, NQO-1 and HO-1 genes was strengthened by Ipre in comparison with either the sham-operated or I/R control group $(p<0.05$, Figure 7$)$. Adding the implemented pharmacological agents to Ipre revealed effectiveness in enhancing expression of these genes compared to the Ipre group. It was evident that Ipre + levosimendan strengthened the expression of these genes compared to the Ipre + cilostazol treated group ( $p<0.05$, Figure 7$)$.

\section{Discussion}

Renal I/R injury is the consequence of many functional and dynamic processes, in which the 
A

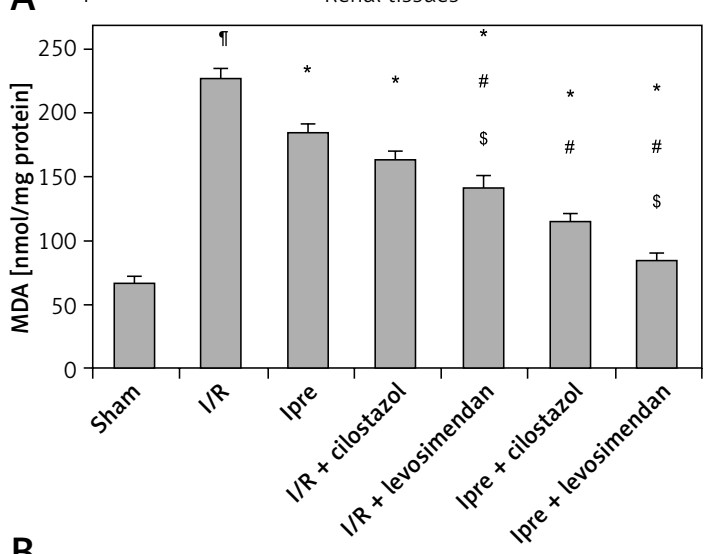

B

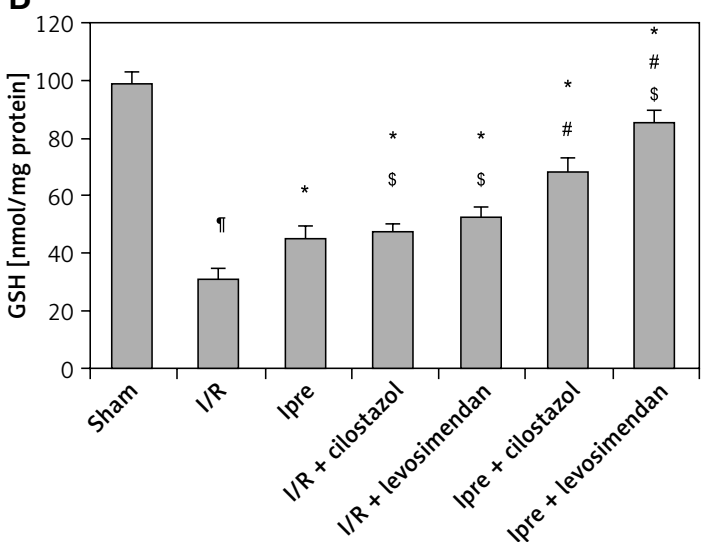

C

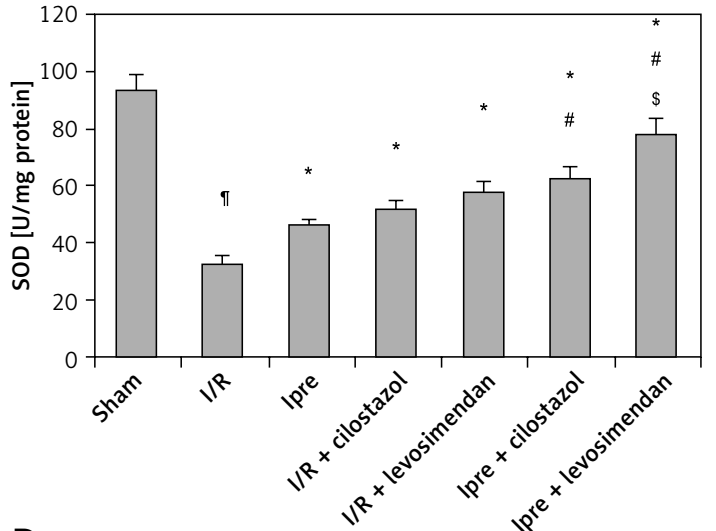

D

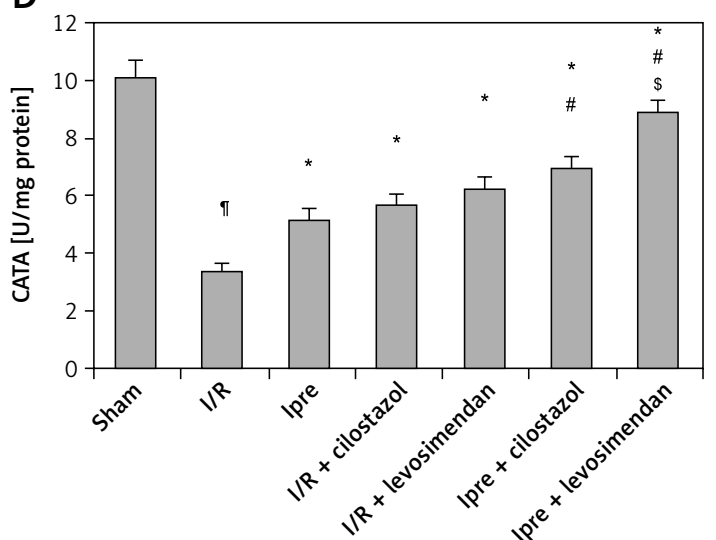

A ॥

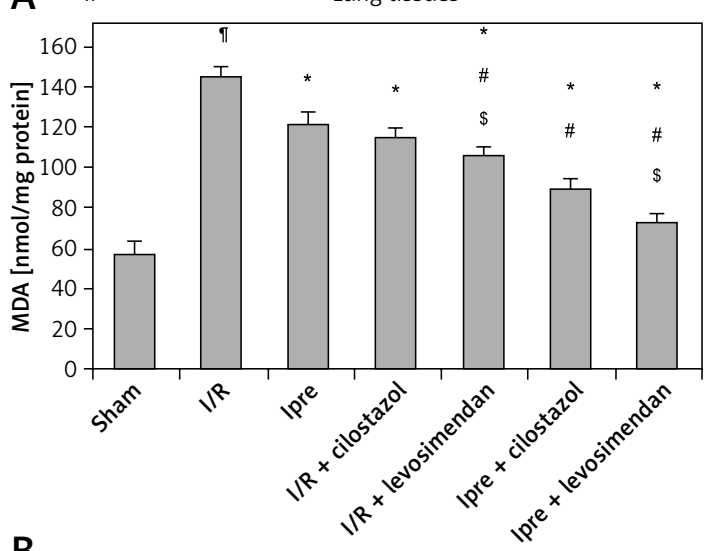

B

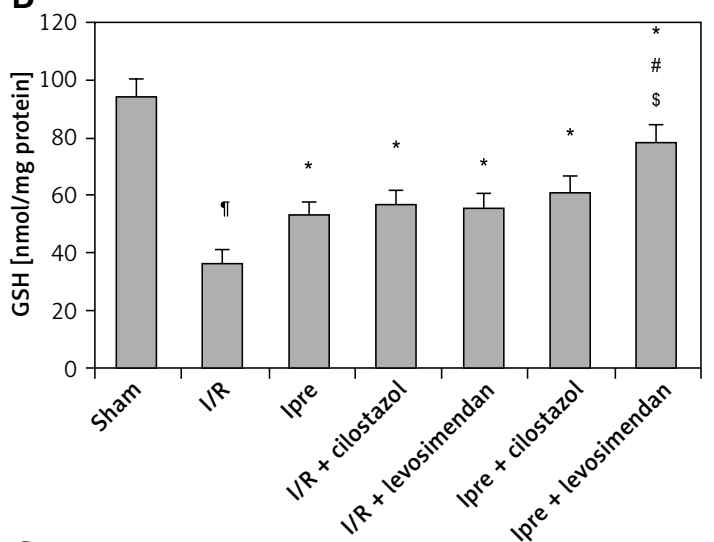

C

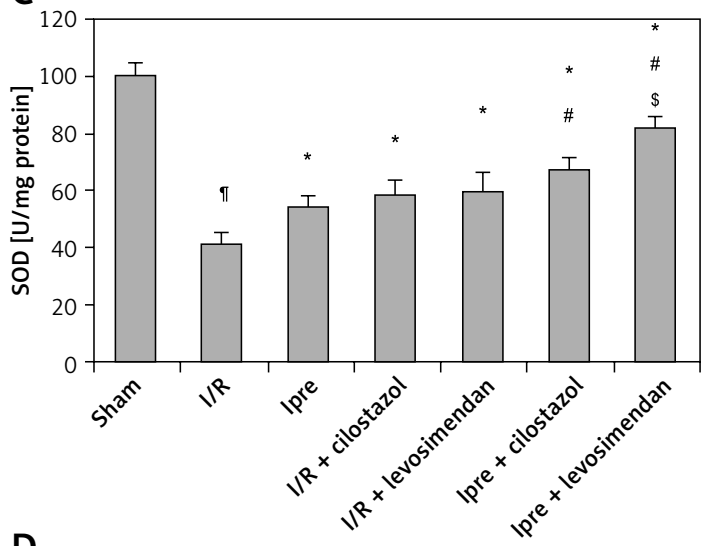

D

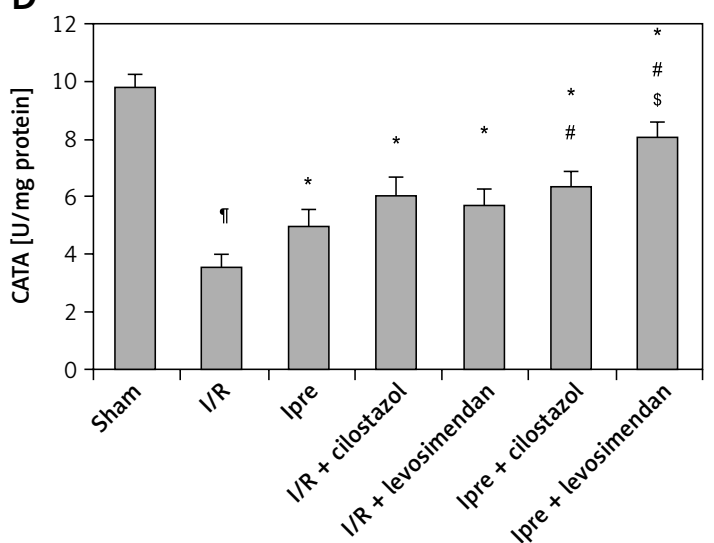

Figure 1. Renal and lung oxidative stress level in the study groups. The effect of ischemic preconditioning (Ipre), ischemia/reperfusion (I/R), cilostazol or levosimendan and combination of them on renal (I) and lung (II) oxidative stress markers, A - MDA, B - GSH, C - SOD, D - CATA. Values are mean \pm SD $(n=8-10)$, analyzed by one-way ANOVA followed by Tukey's multiple comparisons test. "., $\#$, sP $<0.05$. "Compared to sham group. "Compared to I/R group. ${ }^{\# C}$ Compared to Ipre group. ${ }^{5}$ Compared to Ipre + cilostazol 

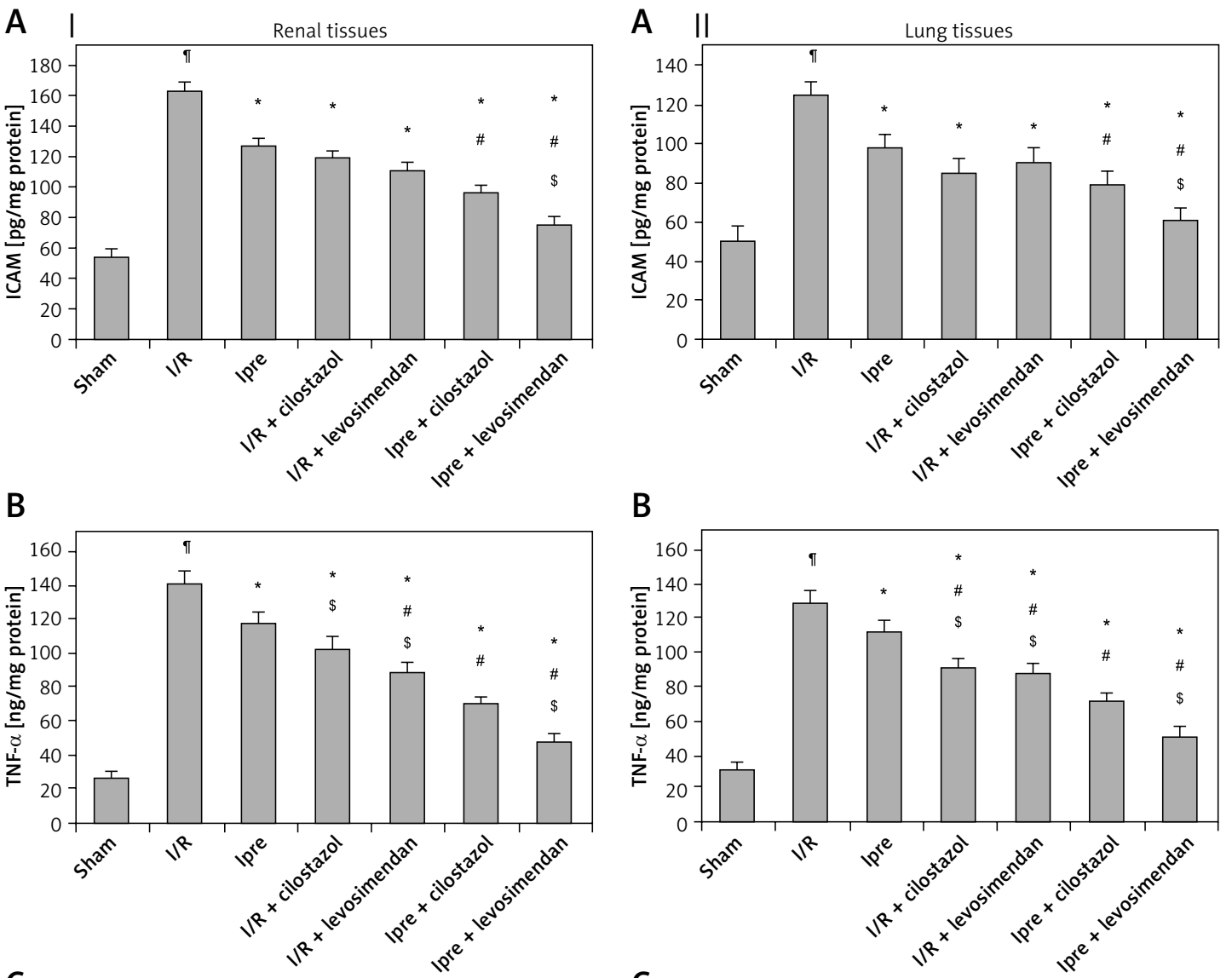

B

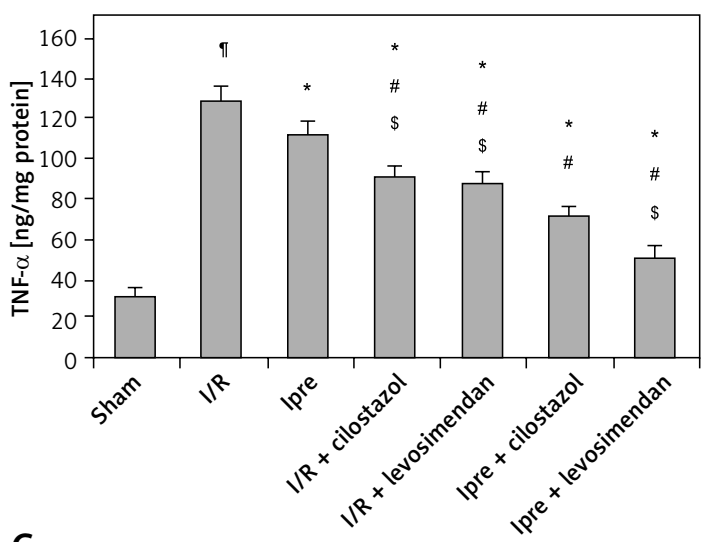

C

C
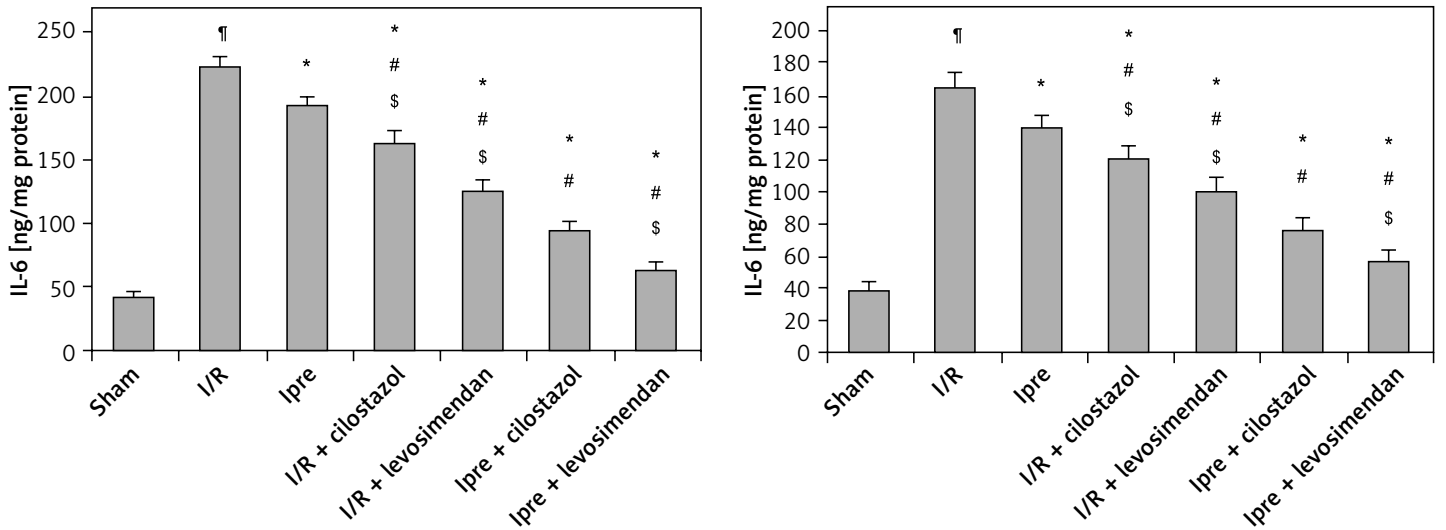

Figure 2. Renal and lung inflammatory markers. The effect of ischemic preconditioning (Ipre), ischemia/reperfusion (I/R), cilostazol or levosimendan on renal (I) and lung (II) tissues, A - ICAM, B - TNF- $\alpha, \mathbf{C}-$ IL-6. Values are mean \pm SD $(n=8-10)$, analyzed by one-way ANOVA followed by Tukey's multiple comparisons test. "I, , \#, \$P<0.05. "Com-

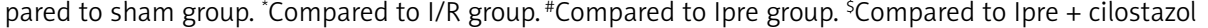

underlying pathophysiological mechanisms revolve around oxidative stress that starts in the kidney and ends in lung inflammation [34, 35]. In contrast, Ipre has a renal protective effect [8], which protects the kidney by activating the antioxidant Nrf2 defense gene expression [33]. Augmentation of Ipre by levosimendan and cilostazol provided a further step in renal and lung protection against I/R.

Figure 8 summarizes the findings as follows: Ipre combination with cilostazol or levosimendan was associated with upregulated expression of renal antioxidant genes (Nrf2/HO-1/NQO-1). This is associated with a decrease in renal apoptosis and oxidative stress burden and an improvement in renal and lung histopathological and functional parameters.

Ischemia/reperfusion injury associated with the restoration of blood flow and re-oxygenation [10] initiates ROS and pro-inflammatory cytokine production $[2,6,9,34]$. The phenomenon known as 

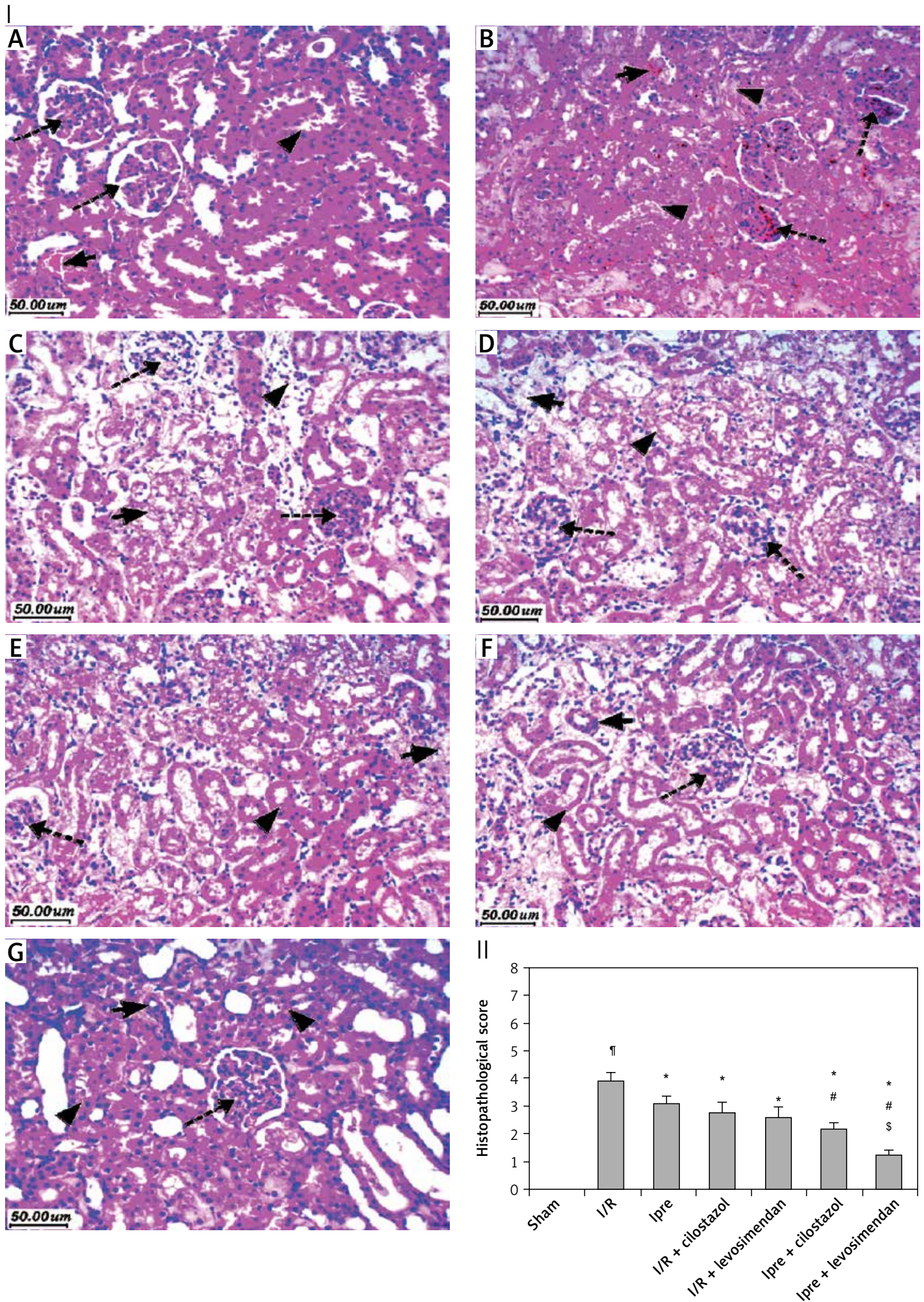

Figure 3. Microphotographs of renal tissues of sham, ischemia/reperfusion (I/R), ischemic preconditioning (Ipre), cilostazol or levosimendan groups (H\&E 400x). I: Sham (A) showed normal glomeruli and tubular cells (dashed arrows). I/R group (B) showed marked interstitial hemorrhage (arrow), tubular epithelial degeneration (arrowhead) and shrunken glomeruli (dashed arrow) with moderate inflammatory infiltrate. Ipre group (C), I/R + cilostazol (D) and $\mathrm{I} / \mathrm{R}+$ levosimendan $(\mathrm{E})$ groups showed mild improvement. Ipre + cilostazol treated group $(\mathrm{F})$ showed moderate improvement. Ipre + levosimendan treated group (G) showed preserved renal architecture. II. Histopathological score. Values are mean \pm SD $(n=8-10)$, analyzed by one-way ANOVA followed by Tukey's multiple comparisons

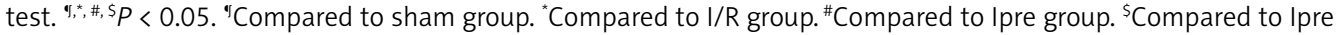
+ cilostazol 

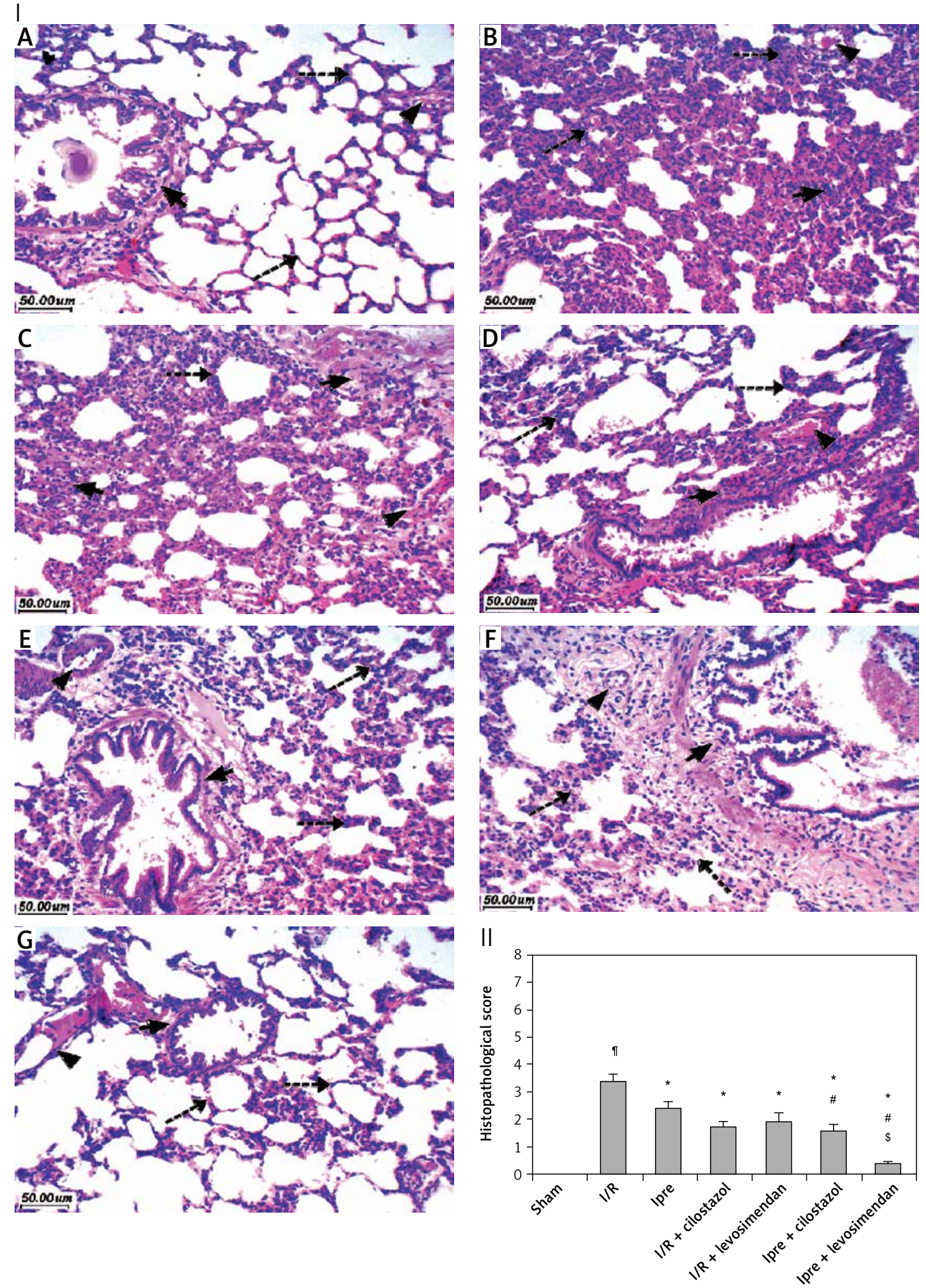

Figure 4. Microphotographs of lung tissues of renal sham, ischemia/reperfusion (I/R), ischemic preconditioning (Ipre), cilostazol or levosimendan groups (H\&E 400x). I: Sham (A) showed preserved lung alveoli (dashed arrow), thin walled vessels (arrowhead) and thin bronchioles (arrow) wall. Renal I/R group (B) showed disturbed lung architecture with marked inflammatory infiltrate and congestion (arrowhead), bronchiolar wall thickening. Rats' lungs of Ipre group (C), I/R + cilostazol (D) and I/R + levosimendan (E) groups showed mild improvement. Ipre + cilostazol treated group (F) showed moderate improvement. Levosimendan with Ipre induced marked improvement. II: Lung histopathological score in renal I/R injury. Values are mean \pm SD $(n=8-10)$, analyzed by one-way ANOVA followed by Tukey's multiple comparisons test. ",",\#, $\mathrm{s} P<0.05$. "Compared to sham group. "Compared to I/R group. \#Compared to Ipre group. ${ }^{\$}$ Compared to Ipre + cilostazol 

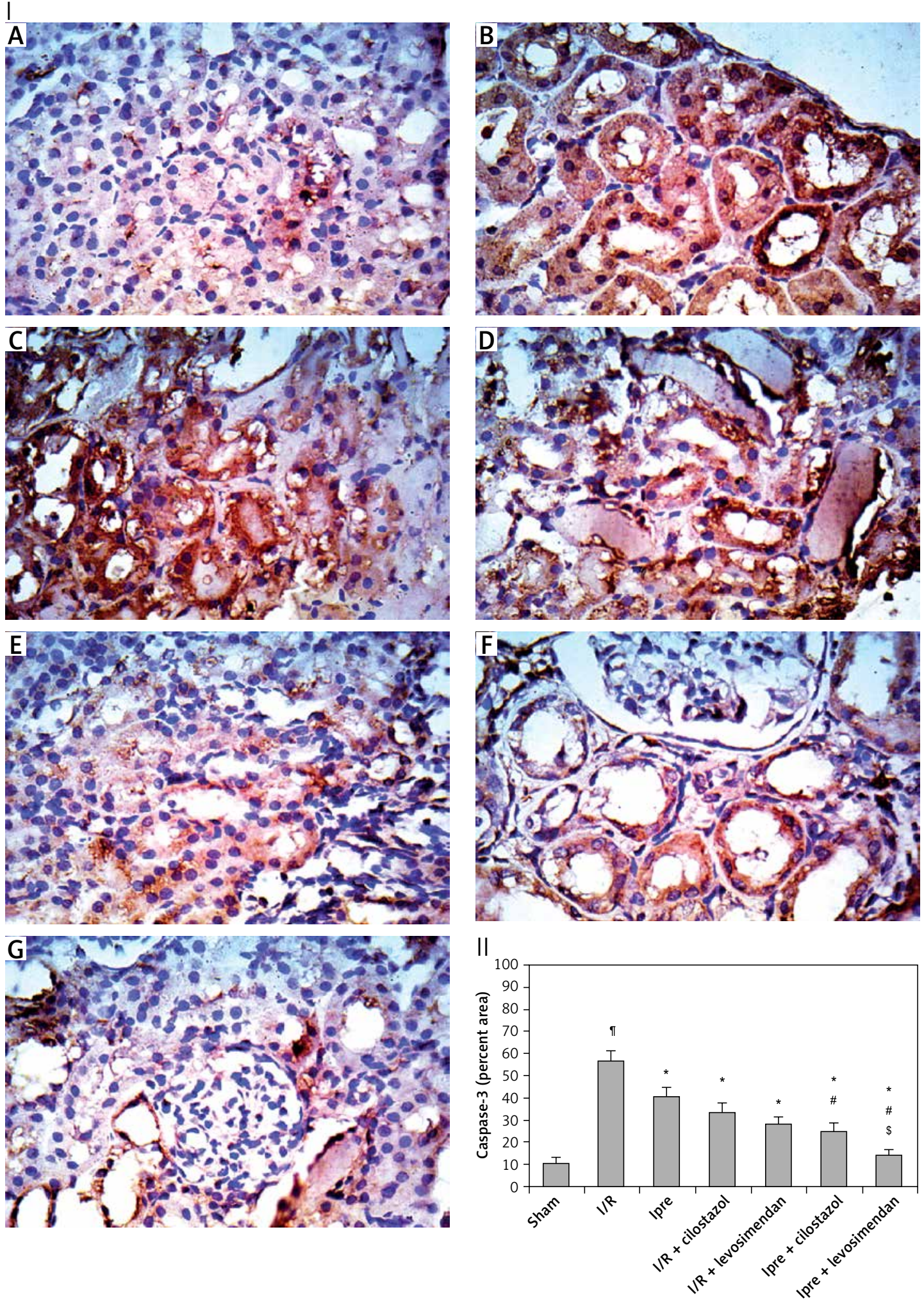

Figure 5. Renal caspase-3 stain microphotographs in different groups (400x). I, A - Sham, B - ischemia/reperfusion (I/R), C - ischemic preconditioning (Ipre), D - I/R + cilostazol, E - I/R + levosimendan, F - Ipre + cilostazol, G - Ipre + levosimendan. II: Values are mean \pm SD $(n=8-10)$, analyzed by one-way ANOVA followed by Tukey's multiple comparisons test. "., \#, $\$ P<0.05$. "Compared to sham group. "Compared to I/R group. "Compared to Ipre group. ${ }^{s}$ Compared to Ipre + cilostazol 

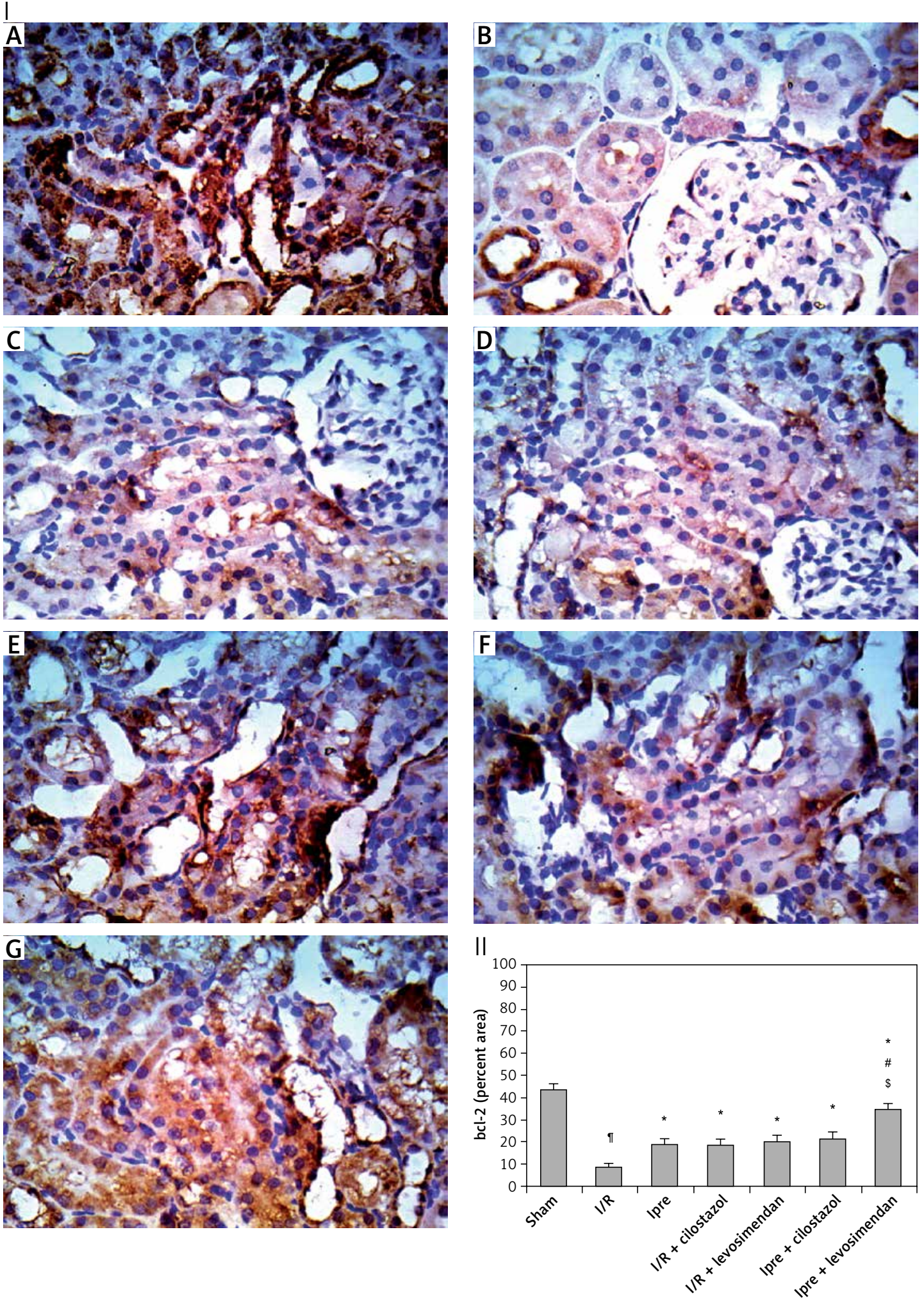

Figure 6. Renal Bcl-2 stain microphotographs in different groups (400x). I, A - Sham, B - ischemia/reperfusion (I/R), C - ischemic preconditioning (Ipre), D - I/R + cilostazol, E - I/R + levosimendan, F - Ipre + cilostazol, G - Ipre + levosimendan. II: Values are mean \pm SD $(n=8-10)$, analyzed by one-way ANOVA followed by Tukey's multiple comparisons test. "., \#, $\$ P<0.05$. "Compared to sham group. "Compared to I/R group. "Compared to Ipre group. ${ }^{\text {s}}$ Compared to Ipre + cilostazol 
A

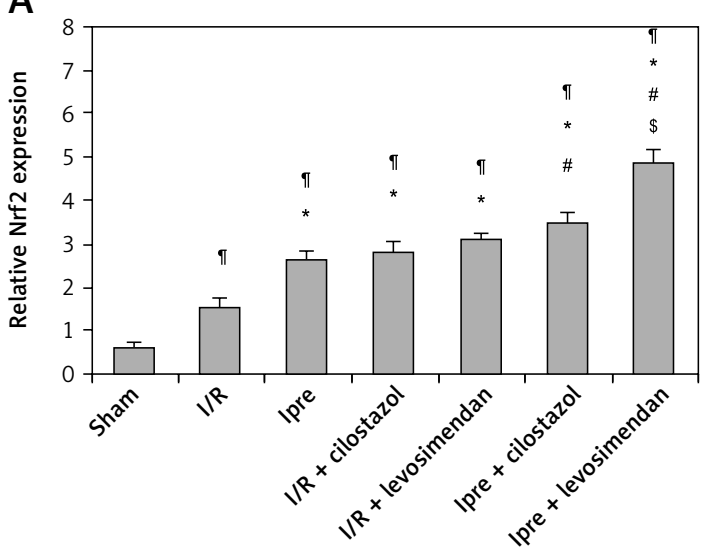

C

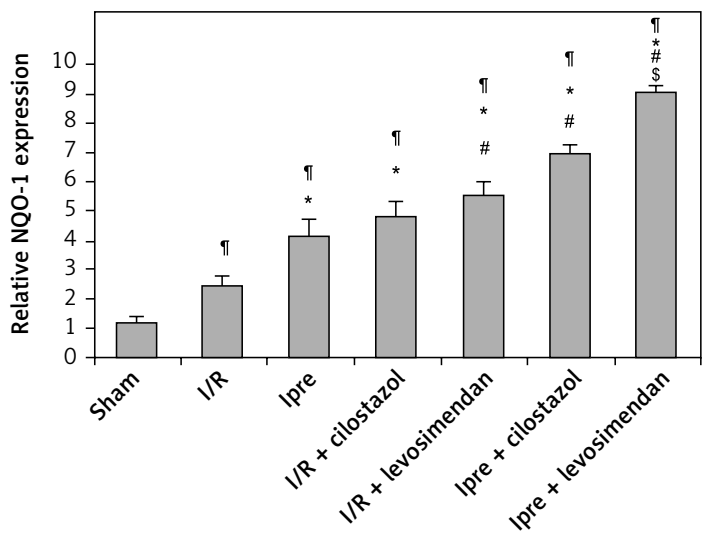

Ipre was shown to enhance renal cellular resistance to ischemia after hypoxic preconditioning [36]. Different Ipre models are proved to partially protect the kidney against I/R injury $[8,10,33]$. However, both cilostazol and levosimendan, by their antiplatelet and anti-inflammatory properties [22, 26, 37-39], enhanced Ipre- induced renal and lung protection, revealed by the improvement of the renal and lung functional and structural parameters.

Local renal I/R injury proved to have deleterious effects on the lung [40], as the current study confirmed. Therefore, in the I/R group, acidosis and impaired oxygenation were detected [32]. This deleterious effect was improved with Ipre and augmented by cilostazol or levosimendan via their ameliorating effect on the lung inflammation and oxidative stress as was evident in the current study and previous studies [34, 35]. The released renal inflammatory cytokines affecting the lung $[1,4,41]$ were ameliorated by I pre with cilostazol or levosimendan through their systemic anti-inflammatory and antioxidant effects [17, 19, $24,38,42]$. Renal ischemia induces endothelial damage with endothelial-leukocyte cell adhesion, causing blockage in microvascular blood flow [2] and an increase in the ICAM protein expression, as evident in the present results, which was reduced with Ipre, cilostazol or levosimendan treat-

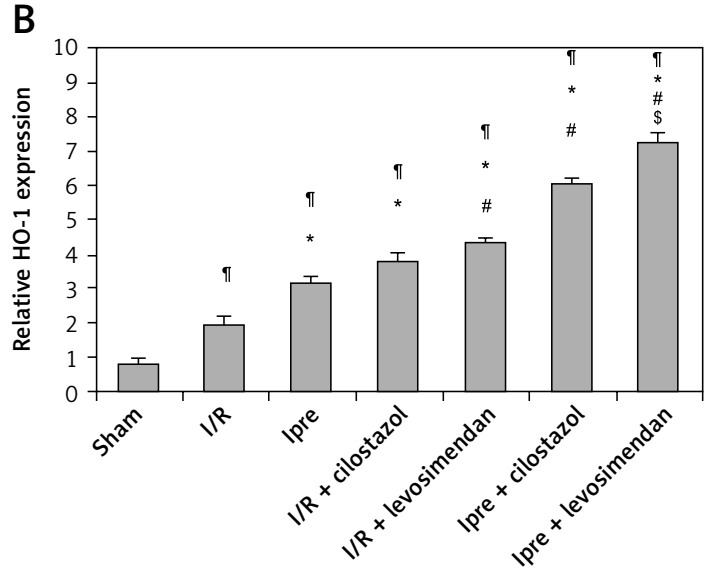

Figure 7. Renal expression of $\mathrm{Nrf} 2 / \mathrm{HO}-1 / \mathrm{NQO}-1$ assessed by PCRin differentgroups.A-Nrf2,B-HO-1, C - NQO-1. Values are mean \pm SD $(n=8-10)$, analyzed by one-way ANOVA followed by Tukey's multiple comparisons test. "., $\#, \$ P<0.05$. "Compared to sham group. * Compared to ischemia/reperfusion (I/R) group. "Compared to ischemic preconditioning (Ipre) group. ${ }^{\$}$ Compared to Ipre + cilostazol

ment. ICAM upregulation is a key marker for the endothelial-leucocyte adhesion processes [24], releasing the pro-inflammatory cytokines TNF $\alpha$ and IL-6, which supports the present observations [2]. The released cytokines enhance leukocyte sequestration [43], which yield more renal injury through ROS production [44]. The present study also revealed similar changes in the pulmonary ICAM expression. Released renal inflammatory mediators could affect pulmonary microvascular endothelial cells, which are a potential link between the kidney and lung injury [32, 34]. Cilostazol and levosimendan have been shown to improve the inflammatory and oxidative stress burden in heart and lung tissues [24, 45-47]. Therefore, AKI ramification on kidney and lung can be attenuated by modulation of the I/R-induced inflammatory response.

Ipre augmented by cilostazol or levosimendan decreased renal apoptosis in response to I/R injury $[24,39]$, thus favoring cell survival. Moreover, Ipre is associated with stimulation of the key regulator of the Nrf2 antioxidant defense gene and its two related antioxidant genes, HO-1 and NQO-1 [12, 17, 48]. Ipre augmented-Nrf2/HO-1/NQO-1 expression, which was enhanced by Ipre + levosimendan or cilostazol, playing a major role in the detoxification of ROS [48]. The upregulation of the antioxidant gene battery has therapeutic and clinical 


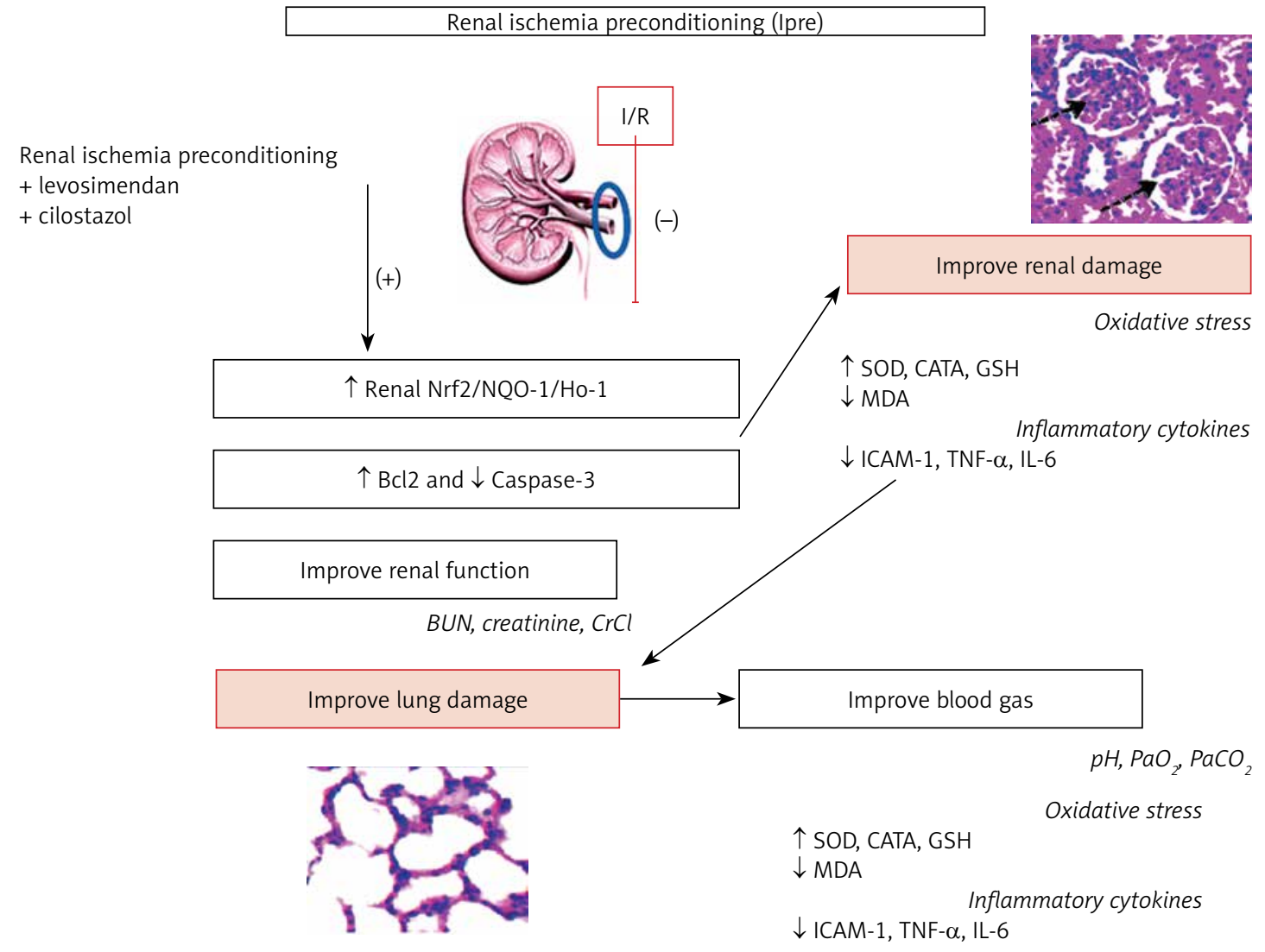

Figure 8. Schematic representation of ischemic preconditioning (Ipre) pathophysiology and its augmentation by cilostazol or levosimendan. Summary of the ROS, inflammatory and gene expression changes are shown, with the protective effect of Ipre combined with cilostazol or levosimendan on renal ischemia/reperfusion (I/R) injury

applications [49], and enhances understanding the pathophysiology of renal I/R [50].

In conclusion, cilostazol and levosimendan enhanced the renoprotective effects of Ipre against acute renal I/R injury. The fundamental mechanisms underlying the positive influence of Ipre and drug treatment might be due to activation of the endogenous adaptive renoprotective gene $\mathrm{Nrf2}$, and its dependent antioxidant genes $\mathrm{HO}-1$ and NOQ1, with suppression of oxidative stress, inflammatory response, and apoptotic cell death. The summation of these processes has local protective effects on the kidney and remote effects on the lung. This helps in understanding the pathophysiological mechanisms associated with I/R and the key role of cilostazol and levosimendan in treatment of acute and chronic renal diseases.

\section{Acknowledgments}

Prof. Laila Rashed, Biochemistry Department, Faculty of Medicine, KasrAini University.

Dr. Mohammed Kamal, Pathology Department, Faculty of Medicine, Suez Canal University.

\section{Conflict of interest}

The authors declare no conflict of interest.

\section{References}

1. Liu F, Ni W, Zhang J, Wang G, Li F, Ren W. Administration of curcumin protects kidney tubules against renal ischemiareperfusion injury (RIRI) by modulating nitric oxide (NO) signaling pathway. Cell Physiol Biochem 2017; 44: 401-11.

2. Bonventre JV, Zuk A. Ischemic acute renal failure: an inflammatory disease? Kidney Int 2004; 66: 480-5.

3. Munshi R, Hsu C, Himmelfarb J. Advances in understanding ischemic acute kidney injury. BMC Med 2011; 9: 11.

4. Andres-Hernando A, Okamura K, Bhargava R, et al. Circulating IL-6 upregulates IL-10 production in splenic CD4+ T cells and limits acute kidney injury-induced lung inflammation. Kidney Int 2017; 91: 1057-69.

5. Makary S, Abdo M, Hassan WA, Tawfik MK. Angiotensin blockade attenuates diabetic nephropathy in hypogonadal adult male rats. Can J Physiol Pharmacol 2019; 97: 708-20.

6. Tong F, Zhou X. The Nrf2/HO-1 pathway mediates the antagonist effect of $L$-arginine on renal ischemia/reperfusion injury in rats. Kidney Blood Press Res 2017; 42: 519-29.

7. Azarkish F, Nematbakhsh M, Fazilati M, et al. N-acetylcysteine prevents kidney and lung disturbances in renal ischemia/reperfusion injury in rat. Int J Prev Med 2013; 4: 1139-46.

8. Shen S, Zhou J, Meng S, et al. The protective effects of ischemic preconditioning on rats with renal ischemiareperfusion injury and the effects on the expression of Bcl-2 and Bax. Exp Therap Med 2017; 14: 4077-82. 
9. Khalid U, Jenkins RH, Pino-Chavez G, Bowen T, Fraser DJ, Chavez R. A localized ischemic preconditioning regimen increases tumor necrosis factor alpha expression in a rat model of kidney ischemia-reperfusion injury. Exp Clin Transplant 2015; 13: 535-42.

10. Joo JD, Kim M, D’Agati VD, Lee HT. Ischemic preconditioning provides both acute and delayed protection against renal ischemia and reperfusion injury in mice. J Am Soc Nephrol 2006; 17: 3115-23.

11. Shokeir AA, Hussein AM, Awadalla A et al. Protection against renal ischaemia/reperfusion injury: a comparative experimental study of the effect of ischaemic preconditioning vs. postconditioning. Arab J Urol 2012; 10: 418-24.

12. Shokeir AA, Hussein AM, Barakat N, Abdelaziz A, Elgarba $M$, Awadalla A. Activation of nuclear factor erythroid 2-related factor 2 (Nrf2) and Nrf-2-dependent genes by ischaemic pre-conditioning and post-conditioning: new adaptive endogenous protective responses against renal ischaemia/reperfusion injury. Acta Physiol (Oxf) 2014; 210: 342-53.

13. Cheng J, Wang H, Zhang Z, Liang K. Stilbene glycoside protects osteoblasts against oxidative damage via Nrf2/ HO-1 and NF-kappaB signaling pathways. Arch Med Sci 2019; 15: 196-203.

14. Ruiz S, Pergola PE, Zager RA, Vaziri ND. Targeting the transcription factor Nrf2 to ameliorate oxidative stress and inflammation in chronic kidney disease. Kidney Int 2013; 83: 1029-41.

15. Duan Q, Sun W, Yuan H, Mu X. MicroRNA-135b-5p prevents oxygen-glucose deprivation and reoxygenation-induced neuronal injury through regulation of the GSK-3beta/Nrf2/ARE signaling pathway. Arch Med Sci 2018; 14: 735-44.

16. Qiao YQ, Jiang PF, Gao YZ. Lutein prevents osteoarthritis through Nrf2 activation and downregulation of inflam mation. Arch Med Sci 2018; 14: 617-24.

17. Park JH, Choi BH, Ku SK, et al. Amelioration of high fat diet-induced nephropathy by cilostazol and rosuvastatin. Arch Pharm Res 2017; 40: 391-402.

18. Shen J, Rasmussen M, Dong QR, Tepel M, Scholze A. Expression of the NRF2 target gene NQO1 is enhanced in mononuclear cells in human chronic kidney disease. Oxid Med Cell Longev 2017; 2017: 9091879.

19. Gokce M, Yuzbasioglu MF, Bulbuloglu E, et al. Cilostazol and diltiazem attenuate cyclosporine-induced nephrotoxicity in rats. Transplant Proc 2012; 44: 1738-42.

20. Sahin MA, Onan B, Guler A, et al. Cilostazol, a type III phosphodiesterase inhibitor, reduces ischemia/reperfusion-induced spinal cord injury. Heart Surg Forum 2011; 14: E171-7.

21. Lee JH, Oh GT, Park SY, et al. Cilostazol reduces atherosclerosis by inhibition of superoxide and tumor necrosis factor-alpha formation in low-density lipoprotein receptor-null mice fed high cholesterol. J Pharmacol Exp Ther 2005; 313: 502-9.

22. Tawfik MK, El-Kherbetawy MK, Makary S. Cardioprotective and anti-aggregatory effects of levosimendan on isoproterenol-induced myocardial injury in high-fatfed rats involves modulation of PI3K/Akt/mTOR signaling pathway and inhibition of apoptosis: comparison to cilostazol. J Cardiovasc Pharmacol Ther 2018; 23 456-71.

23. Zhou C, Gong J, Chen D, Wang W, Liu M, Liu B. Levosimendan for prevention of acute kidney injury after cardiac surgery: a meta-analysis of randomized controlled trials. Am J Kidney Dis 2016; 67: 408-16.
24. Krychtiuk KA, Watzke L, Kaun C, et al. Levosimendan exerts anti-inflammatory effects on cardiac myocytes and endothelial cells in vitro. Thromb Haemost 2015; 113: 350-62.

25. Yakut N, Yasa H, Bahriye Lafci B, et al. The influence of levosimendan and iloprost on renal ischemia-reperfusion: an experimental study. Interact Cardiovasc Thorac Surg 2008; $7:$ 235-9.

26. Grossini E, Molinari C, Pollesello P, et al. Levosimendan protection against kidney ischemia/reperfusion injuries in anesthetized pigs. J Pharmacol Exp Ther 2012; 342: 376-88.

27. Ragab D, Abdallah DM, El-Abhar HS. Cilostazol renoprotective effect: modulation of PPAR-gamma, NGAL, KIM-1 and IL-18 underlies its novel effect in a model of ischemia-reperfusion. PLoS One 2014; 9: e95313.

28. Tawfik MK. Renoprotective activity of telmisartan versus pioglitazone on ischemia/reperfusion induced renal damage in diabetic rats. Eur Rev Med Pharmacol Sci 2012; 16: 600-9.

29. Arslan M, Comu FM, Alkan M, et al. Effect of levosimendan on erythrocyte deformability during myocardial ischaemia-reperfusion injury. Bratisl Lek Listy 2015; 116: 47-50.

30. Yuzawa I, Yamada M, Fujii K. An oral administration of cilostazol before focal ischemia reduces the infarct volume with delayed cerebral blood flow increase in rats. J Stroke Cerebrovasc Dis 2008; 17: 281-6.

31. Honisch A, Theuring N, Ebner B, Wagner C, Strasser RH, Weinbrenner $C$. Postconditioning with levosimendan reduces the infarct size involving the PI3K pathway and KATP-channel activation but is independent of PDE-III inhibition. Basic Res Cardiol 2010; 105: 155-67.

32. Li J, Chen $\mathrm{O}, \mathrm{He} X$, et al. Dexmedetomidine attenuates lung apoptosis induced by renal ischemia-reperfusion injury through alpha2AR/PI3K/Akt pathway. J Transl Med 2018; 16: 78.

33. Shen X, Hu B, Xu G, et al. Activation of Nrf2/HO-1 pathway by glycogen synthase kinase-3beta inhibition attenuates renal ischemia/reperfusion injury in diabetic rats. Kidney Blood Press Res 2017; 42: 369-78.

34. Tawfik MK, Abo-Elmatty DM, Ahmed AA. The role of ATP-sensitive potassium channel blockers in ischemiareperfusion-induced renal injury versus their effects on cardiac ischemia reperfusion in rats. Eur Rev Med Pharmacol Sci 2009; 13: 81-93.

35. Yeh JH, Yang YC, Wang JC, Wang D, Wang JJ. Curcumin attenuates renal ischemia and reperfusion injury-induced restrictive respiratory insufficiency. Transplant Proc 2013; 45: 3542-5.

36. Murry CE, Jennings RB, Reimer KA. Preconditioning with ischemia: a delay of lethal cell injury in ischemic myocardium. Circulation 1986; 74: 1124-36.

37. Antoniades C, Tousoulis D, Koumallos N, Marinou K, Stefanadis C. Levosimendan: beyond its simple inotropic effect in heart failure. Pharmacol Ther 2007; 114: 184-97.

38. Gecit I, Kavak S, Yuksel MB, et al. Effect of short-term treatment with levosimendan on oxidative stress in renal tissues of rats. Toxicol Ind Health 2014; 30: 47-51.

39. Tsao CM, Li KY, Chen SJ, et al. Levosimendan attenuates multiple organ injury and improves survival in peritonitis-induced septic shock: studies in a rat model. Crit Care 2014; 18: 652.

40. Ravikumar P, Li L, Ye J, et al. alphaKlotho deficiency in acute kidney injury contributes to lung damage. J Appl Physiol (1985) 2016; 120: 723-32.

41. Hoke TS, Douglas IS, Klein CL, et al. Acute renal failure after bilateral nephrectomy is associated with cytokine- 
mediated pulmonary injury. J Am Soc Nephrol 2007; 18: 155-64.

42. Aoki C, Hattori Y, Tomizawa A, Jojima T, Kasai K. Anti-inflammatory role of cilostazol in vascular smooth muscle cells in vitro and in vivo. J Atheroscler Thromb 2010; 17: 503-9.

43. Patschan D, Patschan S, Muller GA. Inflammation and microvasculopathy in renal ischemia reperfusion injury. J Transplant 2012; 2012: 764154.

44. Sutton TA, Fisher CJ, Molitoris BA. Microvascular endothelial injury and dysfunction during ischemic acute renal failure. Kidney Int 2002; 62: 1539-49.

45. Parkkonen J, Hasala H, Moilanen E, Giembycz MA Kankaanranta $\mathrm{H}$. Phosphodiesterase 4 inhibitors delay human eosinophil and neutrophil apoptosis in the absence and presence of salbutamol. Pulm Pharmacol Ther 2008; 21: 499-506.

46. Revermann M, Schloss M, Mieth A, et al. Levosimendan attenuates pulmonary vascular remodeling. Intensive Care Med 2011; 37: 1368-77.

47. Karakus E, Halici Z, Albayrak A, et al. Beneficial pharmacological effects of levosimendan on antioxidant status of acute inflammation induced in paw of rat: involvement in inflammatory mediators. Basic Clin Pharmacol Toxicol 2013; 112: 156-63.

48. Abed DA, Goldstein M, Albanyan H, Jin H, Hu L. Discovery of direct inhibitors of Keap1-Nrf2 protein-protein interaction as potential therapeutic and preventive agents. Acta Pharm Sin B 2015; 5: 285-99.

49. Bolisetty S, Zarjou A, Agarwal A. Heme oxygenase 1 as a therapeutic target in acute kidney injury. Am J Kidney Dis 2017; 69: 531-45.

50. Regner KR, Roman RJ. Role of medullary blood flow in the pathogenesis of renal ischemia-reperfusion injury. Curr Opin Nephrol Hypertens 2012; 21: 33-8. 